\title{
Dynamics of domain growth driven by dipolar interactions in a perpendicularly magnetized ultrathin film
}

\author{
N. Abu-Libdeh and D. Venu:* \\ Dept. of Physics and Astronomy, McMaster University, Hamilton Ontario, Canada
}

(Dated: June 7, 2018)

\begin{abstract}
Measurements of the ac magnetic susceptibility of perpendicularly magnetized Fe/2 ML $\mathrm{Ni} / \mathrm{W}(110)$ ultrathin films show a clear signature of the dynamics of domain growth and domain density changes in the striped domain pattern that this system supports. The susceptibility peak measured at different constant heating rates in the range $0.20 \mathrm{~K} / \mathrm{s} \leq R \leq 0.70 \mathrm{~K} / \mathrm{s}$ shifts to higher temperature as the heating rate is increased. Analysis using a relaxation model demonstrates quantitatively that the dynamics is driven by a non-equilibrium domain density at (nearly) zero field (i.e. by dipole interactions), and that the temperature shift is due to a response time determined by the pinning of local domain wall segments by structural defects. The fundamental time scale for relaxation of the domain density driven by dipole interactions is of order $10^{5}$ times slower than the fundamental time scale for an individual Barkhausen step driven by an applied field. The increase in the fundamental time scale reflects the relative size of dipole and Zeeman energies, and the need for the correlated motion of the many local domain wall segments required to affect domain growth.

PACS numbers:
\end{abstract}

\section{INTRODUCTION}

Ultrathin films with perpendicular anisotropy are an example of a larger class of two dimensional systems where strong, short range attractive interactions and weak, long range dipole repulsive interactions lead to the formation of domain patterns $\frac{1-3}{\underline{\underline{3}}}$ The magnetic domain patterns in these films provide a unique opportunity for the study of one-dimensional domain walls (more properly domain "lines") in a two dimensional magnetic system and to understand the way in which domain wall dynamics and fluctuations determine many of the finite temperature magnetic properties of the film.

The present article outlines a quantitative experimental investigation of the dynamics of magnetic domain growth driven exclusively by long-range dipole interactions. It is, perhaps, surprising that, given the intense interest in these systems over the last few decades, this question has not been addressed quantitatively. The absence of clean experiments is due to the complicated hierarchy of magnetic relaxation processes, spanning a wide range of time scales, that control the dynamics of these systems. At sufficiently low temperature, the films support an ordered stripe domain pattern $\underline{\underline{4}}$ The motion of these existing domain walls, when driven by an applied magnetic field, is controlled by localized pinning of the domain walls at microstructural defects in the film. $\underline{\underline{5}}$ As the temperature of the films is increased, the stripe domain density changes exponentially with temperature through domain growth and creation mechanisms that are driven by long range dipole interactions ${ }^{6-8} \underline{-8}$ Finally, at a sufficiently high temperature domain wall fluctuations can drive a transition to a different domain pattern through the proliferation of topological defects in the pattern itself $:^{\underline{9}}$ In order to study the domain growth dynamics driven by dipole interactions, it is necessary to use a zero (or very low) field technique with a very wide dynamic range. Only then can one identify the entire heirarchy of dynamic processes, isolate the desired mechanism and study it on the appropriate time scale.

Almost all of the existing experimental studies do not meet these requirements because they have used either large magnetic fields or static imaging. Dynamical studies have used large applied magnetic fields either to study a magnetically saturated state very far from equilibrium, $\frac{10}{0}$ or to move domain walls using Zeeman magnetic forces rather than weak internal dipole forces $\frac{11.12}{12}$ Studies of the domain structure often use magnetic microscopy in no applied field, but they are limited to a near-static characterization of the domains $\stackrel{7.9}{\cdot} \mathrm{A}$ very few studies infer dynamical processes qualitatively by noting the disappearance of individual domains in successive static domain images, $\stackrel{6,13}{=}$ but most recognize the dynamic limitations of magnetic microspcopy by relying on static imaging of domain changes as a function of film thickness,,$\underline{\underline{7}-\underline{9}}$ rather than as a function of temperature.

In contrast, measurements of the ac magnetic susceptibility cannot directly observe domain geometry, but do offer access a wide range of time scales using very small magnetic fields that do not overwhelm the dipolar interactions. There are a handful of reports that use ac susceptibility to characterize domain wall dynamics. On a short time scale, a small ac field is used to "wiggle" existing domain walls in order to study domain wall pinning by microstructural defects ${ }^{5,14}$ in the film that act on a fundamental time of $10^{-9} \mathrm{~s}$. On a long time scale, measurements of the susceptibility curve at different slow heating rates, $R \leq 0.1 \mathrm{~K} / \mathrm{s}$ (so that the entire curve is traced in many minutes to hours), have revealed the dynamic processes involved in resolving topological defects in the domain pattern itself as the system undergoes a phase transition from one pattern to another ${ }^{15}$ These collective processes act on a fundamental time of $10^{0} \mathrm{~s}$. The present article reports experiments on $\mathrm{Fe} / 2.0 \mathrm{ML}$ 
$\mathrm{Ni} / \mathrm{W}(110)$ films, and concentrates on the intermediate time scale, where dipole interactions drive changes in the domain density as the temperature is increased. Measurements of the ac susceptibility curve as function of faster heating rates, $R>0.1 \mathrm{~K} / \mathrm{s}$, reveal that the peak of the susceptibility shifts to higher temperature with greater $R$. An activated model of domain growth driven by the non-equilibrium domain density at zero field reproduces these results over a range of heating rates and sample thicknesses using a single value of a single adjustable parameter. This parameter, the characteristic time scale for dipole-driven domain growth in these films, indicates a dynamics which is $\sim 10^{5}$ slower than that for the field-driven motion of existing domain walls, but much faster than that involved in removing pattern defects. Thus, the use of ac susceptibility as a probe of the domain dynamics permits a coherent description of the entire hierarchy of competing processes which determine the evolution of these systems.

The remainder of the article is divided into 4 sections. In section II, quantitative models that describe the domain wall dynamics on different time scales are reviewed and/or developed. Section III presents experimental results of ac susceptibility measurements on ultrathin $\mathrm{Fe} / 2.0 \mathrm{ML} \mathrm{Ni} / \mathrm{W}(110)$ films, and these results are analysed quantitively in section IV. The final section summarizes the findings of these studies.

\section{THEORY}

\section{A. Local movement and pinning of existing domain walls: $\tau_{p}$}

Ultrathin (1-10 atomic layers thick) ferromagnetic films may have a magnetocrystalline anisotropy that favours perpendicular magnetization. In some cases, it is strong enough to overcome demagnetization effects, leaving a small residual perpendicular anisotropy $\underline{\underline{16}}$ The long range magnetic dipole interaction in this geometry is antiferromagnetic and leads to the creation of domains $\frac{17}{17}$ The integration of the dipole interaction over a two dimensional film gives a logarithmic dependence of the energy on the domain density. Inverting this relation yields the equilibrium domain density $\underline{18,19} n_{e q}(T)$ :

$$
n_{e q}(T)=\frac{2}{\pi \ell} \exp \left(-\frac{E_{W}(T)}{\Omega m}-1\right)
$$

In this equation $\ell=\pi \sqrt{\Gamma / \lambda(T)}$ is the domain wall width, and $E_{W}=4 \sqrt{\Gamma \lambda(T)}$ is the domain wall energy per unit length. In these definitions, $\Gamma$ is the domain wall stiffness derived from the nearest neighbour magnetic exchange coupling, and $\lambda(T)$ is the effective perpendicular anisotropy (including contributions from both the crystalline and demagnetization terms). $\Omega$ is a constant that sets the scale of the magnetic dipole energy, and $m$ is the thickness of the film in monolayers. As the temperature is increased, thermal fluctuations renormalize the mean field anisotropy and $\lambda(T)$ is reduced. This causes the domain wall energy to decrease and the domain density increases exponentially with temperature.

The magnetic susceptibility of a domain phase is due to the motion of existing domain walls when a small field applied perpendicular to the surface causes the width of domains with a parallel magnetization to grow by $\delta$, while those with an antiparallel magnetization shrink by $\delta$. If the average domain density is $n$, then the resulting magnetization is 19

$$
M=M_{\text {sat }} n \delta,
$$

where $M_{\text {sat }}$ is the saturation magnetization. In the limit of a small applied field, the equilibrium dc susceptibility is

$$
\chi_{e q}(T)=\frac{4}{\pi d n_{e q}(T)} \approx A \exp (-\kappa T),
$$

where $d$ is the film thickness, and $A$ and $\kappa$ are phenomenological parameters that have been shown to describe the effect of the exponential increase in domain density in experimental susceptibility data .5 .14

The ac susceptibility measures the oscillation of the domain walls in a small sinusoidal field at angular frequency $\omega$. The response is retarded by pinning of microscopic sections of the domain walls at structural defects with an average binding energy $E_{\text {pin }}$. The response time $\tau_{p}$ can be modelled by an Arrhenius Law with

$$
\tau_{p}(T)=\tau_{0 p} \exp \left(E_{p i n} / k T\right) .
$$

The ac magnetization is then given by a relaxation equation with

$$
\frac{d M(t)}{d t}=\frac{-1}{\tau_{p}(T)}\left(M(t)-\chi_{e q}(T) H(t)\right) .
$$

The steady state solution is

$$
\chi(T)=\frac{1-i \omega \tau_{p}(T)}{1+\omega^{2} \tau_{p}^{2}(T)} \chi_{e q}(T) .
$$

The dynamic prefactor in eq.(6) describes the effect of pinning. The susceptibility decreases at high temperature due to the increase in domain density, and at low temperature due to pinning. A peak situated roughly where $\omega \tau_{p}\left(T_{p i n}\right)=1$ divides regions where the domain walls are pinned or free. The characteristic pinning temperature is therefore

$$
T_{p i n}=\frac{-E_{p i n}}{k \ln \left(\omega \tau_{0 p}\right)} .
$$

Studies have shown that $\tau_{0 p} \approx 10^{-9} \mathrm{~s}$ for this microscopic pinning process. 


\section{B. Removal of topological pattern defects: $\tau_{d}$}

Theoretical ${ }^{19}$ and numerical 20,21 studies have indicated that a phase transition between the striped domain phase and one of a number of delocalized domain phases is expected at a temperature sufficently high that the domain density is large, and domain wall fluctuations are of over-riding importance. The delocalized phases are characterized by the proliferation of topological defects in the stripe domain geometry that break the stripes into segments, and may re-orient the segments, so that positional and/or orientational long range order are lost.

Numerical simulations ${ }^{22,23}$ have suggested that when the system is quenched from a high temperature delocalized phase to the low temperature striped phase, the topological defects persist for a very long time. This is either because macroscopic rearrangements are required,, 22 or because the transition is non-continuous. ${ }^{23} \mathrm{~A}$ recent experimental study of $1.5 \mathrm{ML} \mathrm{Fe} / 2 \mathrm{ML} \mathrm{Ni} / \mathrm{W}(110)$ films has observed the relaxation of the topological defects in quenched films indirectly, through measurements of the ac susceptibility. ${ }^{15}$ The films were cooled from high temperature $(360 \mathrm{~K})$ at a rate of $R=-0.10 \mathrm{~K} / \mathrm{s}$, and the susceptibility curve was measured for different constant rates of heating. The whole susceptibility curve was seen to shift to higher temperature when the constant heating rate $R$ at which it was measured, was decreased. The long time scale (many minutes to an hour) on which this occurred, $\tau_{d}(T)$, was identified as the characteristic time for topological defects to relax. For large $R$, the measurement time was much less than $\tau_{d}(T)$, and system retained the defects of the delocalized phase, giving a susceptibility curve with an instrinsically lower peak temperature. For small $R$, the measurement time was many times $\tau_{d}(T)$ and the system relaxed to the equilibrium, striped phase that had an intrinsic peak at higher temperature. Thus the peak temperature decreased with increasing $R$.

A quantitative description of the relaxation of topological defects was provided by

$$
\tau_{d}(T)=\tau_{0 d} \exp \left(E_{d} / k T\right)
$$

where the fundamental time scale $\tau_{0 d}$ was found to be of order $10^{0} \mathrm{~s}$, and $E_{d}$ is the barrier to the removal of the topological defect. The peak temperature as a function of the heating rate was well described by

$$
T_{p k}(R)=T_{0}-\Delta \exp \left(-t_{e f f}(R)\right)
$$

with $t_{\text {eff }}(R)$ the effective number of time constants that have passed during the measurement while heating at rate $R$ from initial temperature $T_{i}$ :

$$
t_{e f f}(R)=\int_{T_{i}}^{T_{p k}(R)} \frac{d T}{R \tau_{d}(T)} .
$$

$T_{0}$ is the peak temperature when the relaxation to the equilibrium stripe phase is complete.

\section{Changes in domain density: $\tau_{n}$}

Since the entire susceptibility curve relaxes along the temperature axis with time constant $\tau_{d}$, the fundamental time scale for changes in domain density driven by dipole interactions must lie between the two extremes set by $\tau_{0 p}$ and $\tau_{0 d}$. It is possible to access this time scale as well, using measurments of the ac susceptibility with small applied fields.

Measuring $\chi(T)$ involves changing the temperature at a rate $R(\mathrm{~K} / \mathrm{s})$. Because structural defects pin the domain walls, it takes time for domains to grow or contract, and the domain wall density $n(T)$ will lag behind the equilibrium value $n_{e q}(T)$ by a relaxation time $\tau_{n}(T)$. In this case, the measured susceptibility will be

$$
\begin{gathered}
\chi(T)=\frac{1-i \omega \tau_{p}(T)}{1+\omega^{2} \tau_{p}^{2}(T)} \chi_{e f f}(T), \\
\chi_{e f f}(T)=\frac{4}{\pi d n(T)},
\end{gathered}
$$

where $n(T)$ is the history-dependent present value of the domain density during heating or cooling. It is determined by a relaxation equation that is developed in Appendix $\mathrm{A}$. The constant rate of temperature change, $R$, is used to remove the explict time dependence from eq. A5, so that the relaxation equation governing changes in domain density is

$$
\frac{d n(T)}{d T}=\frac{-1}{R \alpha \tau_{p}(T)}\left(n(T)-n_{e q}(T)\right) .
$$

An important point in the development of this equation is the relation between the time constant for the field-induced oscillation of existing domain walls, $\tau_{p}(T)$, and the time constant for dipole-induced changes in the domain density, $\tau_{n}(T)$. Since the pinning sites that retard the motion of existing domain walls when a magnetic field is applied also impede the growth of new stripe domains driven by dipolar interactions, the activation energies for the two processes are the same. However, the characteristic times $\tau_{0 n}$ is expected to be different than $\tau_{0 p}$ because of the need for correlated motion of a larger region of domain wall. For instance, whereas the motion of any part of an existing domain wall will affect the response to a magnetic field equivalently, the motion of some regions of the domain wall are much more effective than others in changing the domain density. Changing the domain density at constant magnetization involves a large movement of a small length of domain wall near the end of a domain segment. Finally, in the relaxation approximation, the number of active growth sites is assumed to be proportional to the difference between the present value of the domain density and the equilibrium value, but the value of this proportionality constant is not obvious from first principles. All of these geometric factors are taken into account through an empirical factor $\alpha$ in eq. A5 , so that $\tau_{0 n}=\alpha \tau_{0 p}$ and $\tau_{n}(T)=\alpha \tau_{p}(T)$. 
It is straighforward to solve eq. (13) using the phenomenological expansion for $\chi_{e q}(T)$ in eq.(3). At high temperature the pinning is ineffective, and $n(T) \rightarrow$ $n_{e q}(T)$, whereas at low temperature the pinning is so effective that $d n / d T \rightarrow 0$ and $n(T)$ saturates. $T_{n}$ is a characteristic temperature dividing these two regimes. It can be estimated 24 by setting $\frac{\partial n}{\partial T}=\frac{\partial n_{e q}}{\partial T}$ to give

$$
n(T)=\left(1-R \alpha \kappa \tau_{p}(T)\right) n_{e q}(T) .
$$

When $R \alpha \kappa \tau_{p}\left(T_{n}\right)=1$ the model becomes unphysical because the domain density cannot change quickly enough to maintain equilibrium, and the domain density must saturate. This occurs near the temperature

$$
T_{n}=\frac{-E_{p i n}}{k \ln \left(R \kappa \alpha \tau_{0 p}\right)} .
$$

The ac susceptibility in eq. (11) depends on the relative values of the characteristic temperatures for domain wall motion, $T_{p i n}$, and domain growth, $T_{n}$. If $T_{n}<T_{p i n}$, then pinning will stop the oscillatory motion of the domain walls in the applied ac field, even though the domain density can still change through domain growth. The dynamical factor involving $\omega \tau_{p}$ will cut off the susceptibility, so that it is insensitive to changes in the domain density below $T_{\text {pin }}$. In this case, $\chi_{\text {eff }} \approx \chi_{\text {eq }}$ over the temperature range of the susceptibility peak, and the susceptibility will not depend upon $R$. On the other hand, if $T_{n}>T_{p i n}$, then the saturation of the domain density occurs in a temperature range where the field-induced oscillation of the domain walls is not pinned, and the susceptibility gives a robust signal. The curve shifts to higher temperature with increasing $R$, since increasing $R$ increases $T_{n}$ in eq.(15). (Note that the direction of this shift is opposite to that described in the previous section for the relaxation of topological pattern defects.) Equating $T_{p i n}$ and $T_{n}$ gives the condition that divides these two behaviours:

$$
\alpha \kappa=\frac{\omega}{R}
$$

Choosing measurement parameters on the right side of this equation such that they are smaller than the combination of physical parameters on the left side, ensures that peak temperature of the susceptibility, $T_{p k}$, is sensitive to the relaxation of the domain density when heating at rate $R$. The experimentally practical range of heating rates is from $0.20 \mathrm{~K} / \mathrm{s}$ (below which the relaxation of topological defects dominates ${ }^{15}$ ), to $1.00 \mathrm{~K} / \mathrm{s}$ (above which the decrease in measurement time at any temperature results in too much noise). A typical value of $\kappa$ is $0.04 K^{-1}$. Choosing $\omega=210 \mathrm{~Hz}$ then gives the minimum value $\alpha_{\min } \approx 10^{4}$ for which the measurement will be sensitive to the relaxation dynamics of the domain density. This magnitude for $\log _{10}\left(\alpha \tau_{0 p}\right)$ is comfortably near the middle of the range between the experimentally determined values of $\log _{10}\left(\tau_{0 p}\right)$ and $\log _{10}\left(\tau_{0 d}\right)$. If the actual value of $\alpha$ is smaller, then the experimental value of $\omega$ can be reduced.
Finally, there is the question of whether or not the size of the shift in $T_{p k}$ as a function of heating rate will be large enough to measure. Appendix B presents an estimate of the expected size. According to eq. (B6), the linear term in the shift of the peak temperature of the susceptibility curve, $T_{p k}$, evaluated locally at $R=R_{0}$, is given by

$$
\frac{\partial T_{p k}}{\partial R} \equiv B \approx \frac{1}{R_{0}\left(\frac{2}{T_{p k}}+\frac{3 E_{p i n}}{k T_{p k}^{2}}+\kappa\right)}
$$

From the measurements in ref.(15) for $1.5 \mathrm{ML}$ Fe/2 ML $\mathrm{Ni} / \mathrm{W}(110)$ films, eq. (17) gives $B \approx+5 \mathrm{~s}$ for $R_{0}=0.5$ $\mathrm{K} / \mathrm{s}$. This small slope is consistent with the data, but cannot be reliably detected. The parameters $E_{p i n}, \kappa$ and $T_{p k}$ all decrease quickly with film thickness. ${ }^{5}$ Films with $2 \mathrm{ML}$ Fe are estimated to have values of $B$ larger by a factor of 2 to 5 . This should provide a clear signature of the dynamics of domain wall growth.

\section{EXPERIMENTAL RESULTS}

Domain dynamics were studied in a series of Fe/2 ML $\mathrm{Ni} / \mathrm{W}(110)$ films. The nickel layer in this structure establishes a slightly strained, epitaxial f.c.c. (111) template on the tungsten substrate, upon which f.c.c. Fe grows epitaxially for a few layers. ${ }^{25}$ Due to the f.c.c. Fe/vacuum interface, this system exhibits perpendicular anisotropy. Above 2.2 ML Fe, the perpendicular anistropy exists only at low temperature and the magnetization becomes inplane at a spin reorientation transition ${ }^{26}$ Below $1.25 \mathrm{ML}$, the magnetic behaviour is complicated by the incomplete formation of the Fe layer. In the present study, the film thickness was restricted to the intermediate range where the description in section II.A has been shown to be valid.

The films were grown in ultrahigh vacuum using thermal evaporation from a evaporator with a calibrated internal flux monitor, following a procedure established in previous studies. ${ }^{25}$ The first monolayer of $\mathrm{Ni}$ was annealed to $600 \mathrm{~K}$ to ensure good wetting and the growth was monitored using Auger electron spectroscopy and low energy electron diffraction. The substrate temperature was measured using a W-Re $5 \% / \mathrm{W}$-Re10\% thermocouple embedded in the $\mathrm{W}$ crystal, and the temperature was controlled by a combination of static cooling through a copper braid attached to a liquid nitrogen reservoir, and active heating by radiation from a filament just beneath the crystal. The rate of heating or cooling could be kept constant to within $0.05 \mathrm{~K} / \mathrm{s}$. The maximum controlled cooling rate across the whole temperature range was $-0.10 \mathrm{~K} / \mathrm{s}$. The maximum useful heating rate was $1.0 \mathrm{~K} / \mathrm{s}$, above which the reduced number of data points introduced excessive noise ${ }^{27}$

The ac magnetic susceptibility was measured using the polar magneto-optic Kerr effect, $\stackrel{28}{2}$ where the rotation of the plane of polarization of linearly polarized light is proportional to the perpendicular component of the magne- 
tization. A current coil near the sample created a sinusoidal ac field of amplitude 2.0 Oe, and the very small polarization rotation from the ultrathin film was detected using a nearly crossed polarizer and lock-in amplification of the output from a photodiode. The ac field had a frequency of $210 \mathrm{~Hz}$ in these studies. The ac current through the heating filament had a much higher frequency, so that it did not interfere with the measurements.

Because the dynamics being studied change the shape of the susceptibility curve and shift it in temperature, considerable effort was invested in developing procedures that removed systematic errors and resulted in reproducible data traces. These included: annealing the films to $400 \mathrm{~K}$ before measurements commenced, and subsequently never heating above $360 \mathrm{~K}$; cooling the sample from $360 \mathrm{~K}$ at a rate of $-0.10 \mathrm{~K} / \mathrm{s}$ before each susceptibility measurement was recorded during heating; discarding the first heating trace to ensure a consistent magnetic history for each measurement; randomizing the order of the measurements for different values of heating rate; demonstrating that the lock-in time constant of $2 \mathrm{~s}$ had no effect on the shape or position of the susceptibility curve for the range of heating rates used.

Figure 1 shows the real part of the susceptibility curves measured for three separate films with Fe thicknesses of 1.5 ML, 1.75 ML and 2.0 ML (all $\pm 0.1 \mathrm{ML}$ ), and heating rates varying from $0.03 \mathrm{~K} / \mathrm{s}$ to $0.70 \mathrm{~K} / \mathrm{s}$. (Note: the data for $1.5 \mathrm{ML} \mathrm{Fe}$ is the same data that is analysed in ref 15). The absolute scale of the susceptibility is uncertain within a factor of about 2 because the magnetooptic Voigt parameter is not well known for these films. However, the relative scale for all traces on all plots is consistently calibrated to the absolute optical rotation. The general shape of the curves is consistent with many previous studies. At high temperature the susceptibility decreases exponentially as the domain density increases. At low temperature the susceptibility decreases exponentially as the domain walls become pinned in structural defects. The peak in the susceptibility occurs at an intermediate temperature due to the interrelation of these two processes, and depends strongly on the film thickness.

The dependence of the susceptibility on the heating rate that is evident in fig. 1 is summarized more clearly in fig. 2 using two quantities: the temperature at the peak of the susceptibility, $T_{p k}$, and the full width at half maximum of the peak. The systematic variation of these quantities with heating rate is the subject of section IV. The remainder of this section is concerned with comparing the variation of the susceptibility with film thickness to previous findings. Quantitative fits to the data, using eq.(6) with parameters defined in eq.(4) and (3), are shown in fig. 3. Part a) demonstrates the quality of the fits and confirms that the essential points of the model are valid. Values of the parameters $E_{\text {pin }}$ and $\kappa$ are given in parts b) and c), respectively. As has been shown previously, 15 the average pinning energy, which is a structural property of each film, is independent of the x ML Fe/2.0 ML NI/W(110)

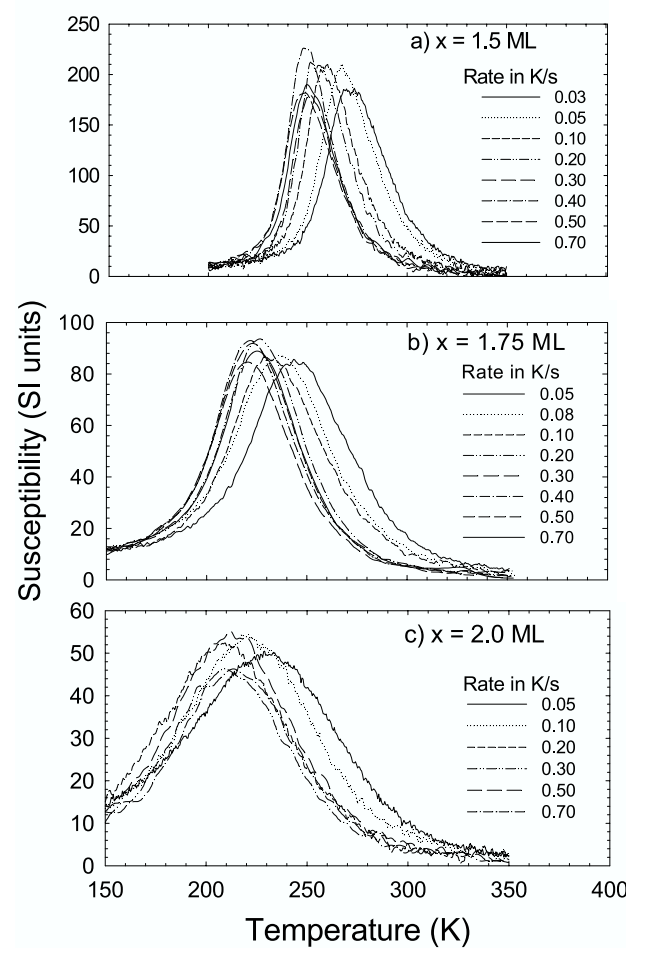

FIG. 1: Magnetic ac susceptibility of $\mathrm{x}$ ML Fe/2 ML $\mathrm{Ni} / \mathrm{W}(110)$ films as a function of temperature for different constant heating rates. All the measurements were performed after cooling the sample from $360 \mathrm{~K}$ at a rate of $-0.10 \mathrm{~K} / \mathrm{s}$. An ac field of 2.0 Oe at a frequency of $210 \mathrm{~Hz}$ was applied normal to the film. Parts a), b) and c) show sequential measurements from a single film of thicknesses 1.5 ML, 1.75 ML and 2.0 ML of Fe, respectively.

heating rate. The pinning energy decreases rapidly with film thickness. A previous study ${ }^{5}$ has shown that the pinning is due to changes in the perpendicular anisotropy caused by changes in the thickness at monolayer steps in the film, and that $E_{p i n} \sim d^{-3 / 2}$ for measurements of a single film grown sequentially to a number of thicknesses. The current results are qualitatively consistent with this finding, but quantitative comparisons are not possible among a series of independently grown films with unrelated microstructure. The parameter $\kappa$ depends on both film thickness and the heating rate. The variation with thickness that displaces the curves one from another in fig. $3 c$ is in qualitative agreement with eq. (3). Because the surface anisotropy varies as $1 / d, \kappa$ can be ultimately derived from eq.(1) as $\kappa \approx \frac{1}{d} \frac{\partial E_{W}}{\partial T} \sim d^{-3 / 2}$.

The thickness dependence of $E_{\text {pin }}$ and $\kappa$ leads to the systematic displacement of the susceptibility curves with thickness observed in fig. 2. A gross measure of $T_{p k}$ is the pinning temperature $T_{p i n}$, and eq.(7) indicates that this scales with $E_{\text {pin }}$. Thus the curves in fig. 2a are displaced to lower temperature for thicker films. Similarly, since 
x ML Fe/2 ML Ni/W(110)

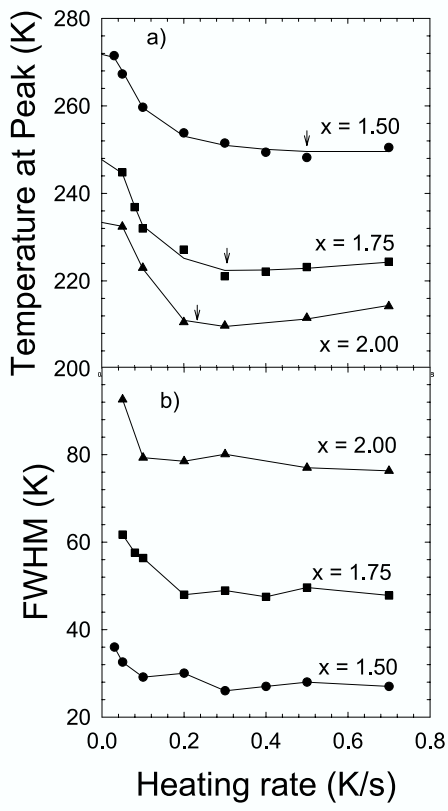

FIG. 2: a) The temperature at the peak of the susceptibility curve, $T_{p k}$, as a function of the heating rate, for films of different Fe thickness. The arrows mark approximately the heating rate at which $T_{p k}$ has a minimum. The fitted lines are discussed in section IV. b) The full-width at half-maximum (FWHM) of the susceptibility curves as a function of heating rate. Lines simply connect the points.

the two exponential factors $\left(E_{p i n}\right.$ and $\left.\kappa\right)$ that cut off the susceptibility above and below its peak value vary inversely with a power of the thickness, the peak width in fig. $2 \mathrm{~b}$ increases as the film thickness increases.

\section{ANALYSIS AND DISCUSSION}

As the heating rate increases, $T_{p k}$ first decreases sharply, and then reverses and gradually increases. The value of $R$ at which this reversal occurs (marked by the arrows in fig. 2a depends systematically on the film thickness. This suggests that there are two competing dynamical processes - one that dominates at small $R$ and the other that dominates at large $R$. It is important to note that the changes in the peak amplitude with $R$ in fig. 1 are very modest even as the peak temperature changes substantially.

The rapid decrease in $T_{p k}$ and in the peak width at small $R$ are correlated to an increase in $\kappa$ in fig. 3. This behaviour was analysed in ref. 15, where it was related to

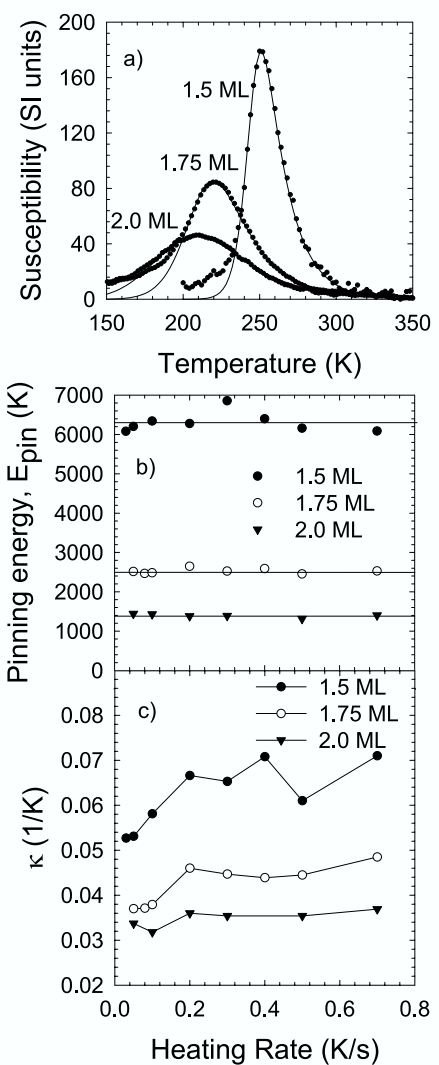

FIG. 3: Fits to the data using the phenomenological parameters from eq. (6). a) Representative fits for measurements at a heating rate of $0.30 \mathrm{~K} / \mathrm{s}$ for different film thicknesses. b) The pinning energy for local Barkhausen steps of microscopic segments of a domain wall. c) The paramter $\kappa$, which reflects the exponential increase in domain density with temperature. Lines simply connect the points.

the slow relaxation of topological defects in the domain pattern after the sample is quenched from a high temperature, delocalized phase, as in section IIB. The present data now confirms this observation at other thicknesses. The second, less dramatic, process at large $R$ is more obvious in the new data at thicknesses of 1.75 and $2.00 \mathrm{ML}$. According to the analysis in section IIC, the increase in $T_{p k}$ at large values of $R$ is qualitatively consistent with the dynamics of domain density changes and growth on an intermediate time scale. This agreement suggests a quantitative analysis using a combination of eq.(9) and (17):

$$
T_{p k}(R)=T_{0}-\Delta \exp \left(-t_{e f f}(R)\right)+B R .
$$

Figure 4 presents the least-squares residuals of the fit of the experimental peak temperatures to this expression by varying $T_{0}, \Delta, E_{d}$ and $B$ for a range of values of the time constant $\tau_{0 d}$. A consistent optimum value of $\log _{10} \tau_{0 d}=0.00 \pm 0.05$ is found. Table I gives the best fit parameters for each film thickness. Error estimates on these parameters, as well as on $\tau_{0 d}$, are derived by hold- 


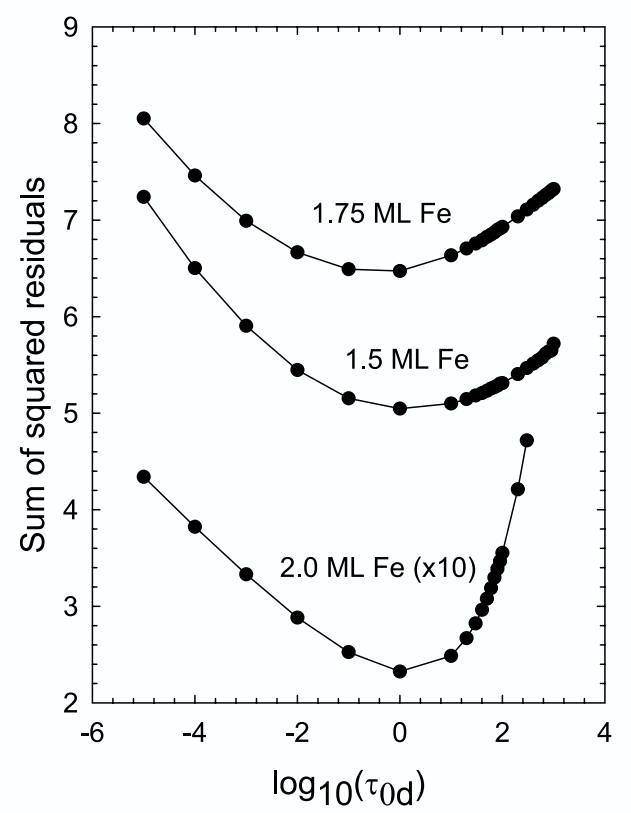

FIG. 4: The sum of squared residuals for the independent fits of eq.18) to the peak temperatures as a function of heating rate in fig. 2a. The residuals for the film with $2.0 \mathrm{ML}$ Fe are multiplied by 10 to place them on the same plot. The residuals for this film are smaller because there are data points at fewer heating rates.

\begin{tabular}{|c|c|c|c|c||c|}
\hline Fe ML & $E_{d}(\mathrm{~K})$ & $T_{0}(\mathrm{~K})$ & $\Delta(\mathrm{K})$ & $B$ fit (s) & $B$ calc. (s) \\
\hline 1.50 & $1560 \pm 25$ & $271.8 \pm 0.6$ & $26.0 \pm 0.9$ & $2.7 \pm 1.8$ & $3.95 \pm 0.5$ \\
\hline 1.75 & $1390 \pm 13$ & $247.7 \pm 0.5$ & $33.8 \pm 0.8$ & $11.0 \pm 1.5$ & $8.91 \pm 0.5$ \\
\hline 2.00 & $1270 \pm 12$ & $234.1 \pm) .5$ & $38.1 \pm 0.7$ & $21.0 \pm 1.5$ & $16.1 \pm 0.5$ \\
\hline
\end{tabular}

TABLE I: Parameters for fitting the data in fig. 2a to eq.(18) appear to the left of the double line. In all cases $\log _{10}\left(\tau_{0 d}\right)=$ $0.00 \pm 0.05$. The parameter $B$ calc. to the right of the double line is determined in fig. 5 .

ing all the other parameters constant, and finding the range of variation that changes the squared residuals by 1.0. The fitted curves are superimposed upon the data in fig. 2a.

These results confirm the earlier analysis of the data for the $1.5 \mathrm{ML}$ Fe films $\stackrel{15}{=}$ where the term $B R$ due to to the dynamics of domain density changes was neglected. Since $B$ is found to be small for this thickness, the values of the other parameters are essentially unchanged from those found in the previous study. The new data for thicker films show that, upon quenching from high temperature, the relaxation parameter $\tau_{0 d}$ and $E_{d}$ for topological defects in the domain pattern do not depend strongly on the film thickness. It continues to take many minutes to an hour to remove these defects and for the magnetic susceptibility to take the equilibrium curve representing the ordered stripe phase. The fact that $E_{d}$ does not scale with $E_{\text {pin }}$ further substantiates the conclusion that the removal of the topological pattern defects is not limited by microscopic pinning mechanisms, but rather by the low probability that weak dipole interactions will drive the co-ordinated domain wall fluctuations required to make the mesoscopic changes required to remove the defect from the pattern. For all thicknesses, the defect relaxation is reflected as well in a decrease in $\kappa$. As was previously noted $\stackrel{15}{\frac{15}{5}}$ the larger value of $\kappa$ in the presence of topological defects may represent their influence on the non-equilibrium free energy, creating an increase in the magnetic "stiffness" as measured by the susceptibility.

Because the constant $B$ is not small for the 1.75 and 2.00 ML Fe films, the data cannot be reasonably fit without this term. This proves that even as the topological defects are relaxing, a second, faster relaxation mechanism is present in these films. The sign and order of magnitude of the second effect is consistent with the estimates from eq.(17) for relaxation of the domain density, and indicate that the domain density and topological defect density are not strongly coupled, but relax rather independently.

The interpretation of the constant $B$ can be tested quantitatively. Fig. 5 gives an example calculation for the film with 1.75 ML Fe. The parameters $A, \kappa, \tau_{0 p}$ and $E_{\text {pin }}$ are those determined in fig. 3a by the fit to eq.(6) for the data with $R=0.30$. In fig. 5 a the result for $\chi_{e q}(T)$ derived from $A$ and $\kappa$ is given by the dashed line. The same parameters are then used to evaluate $n_{e q}(T)$ and derive $n(T)$ as solutions to eq. (13) for different heating rates. The initial condition for each is the saturated, "frozen" domain density that results from solving eq.(13) as a function of cooling from the equilibrium state at $350 \mathrm{~K}$, at a rate of $R=-0.10 \mathrm{~K} / \mathrm{s}$. Once $n(T)$ is known, $\chi_{e f f}(T)$ is easily derived from eq.(12). The solid curves in fig. 5 a give $\chi_{\text {eff }}(T)$ for heating rates of $0.20,0.40$, and $0.70 \mathrm{~K} / \mathrm{s}$ (moving from left to right in the figure), for the choice $\alpha=10^{5.5}$. It is clear that $\chi_{\text {eff }}(T)$ peaks at lower temperature when $R$ is smaller, since the system has more time to relax toward the equilibrium state. Fig. 5b presents $\chi(T)$ by multiplying the results of part a) by the dynamical pre-factor defined in eq.(6) and (11). It can be seen that the calculated susceptibility shifts gradually to higher temperature with higher $R$, but that the amplitude of the peak changes very little.

The position $T_{p k}$ and peak amplitude can be taken from a series of curves such as those in fig. 5b. The linear dependence of these quantities as a function of $R$ can be extracted to give one point on each of fig. $5 \mathrm{c}$ and 5 d. Repeating the calculation for many values of $\alpha$ produces the entire plots. The three traces on these plots correspond to parameters fit to the data in fig. 3a for 
Simulation for $1.75 \mathrm{ML}$ Fe/ 2.0 ML Ni/W(110)
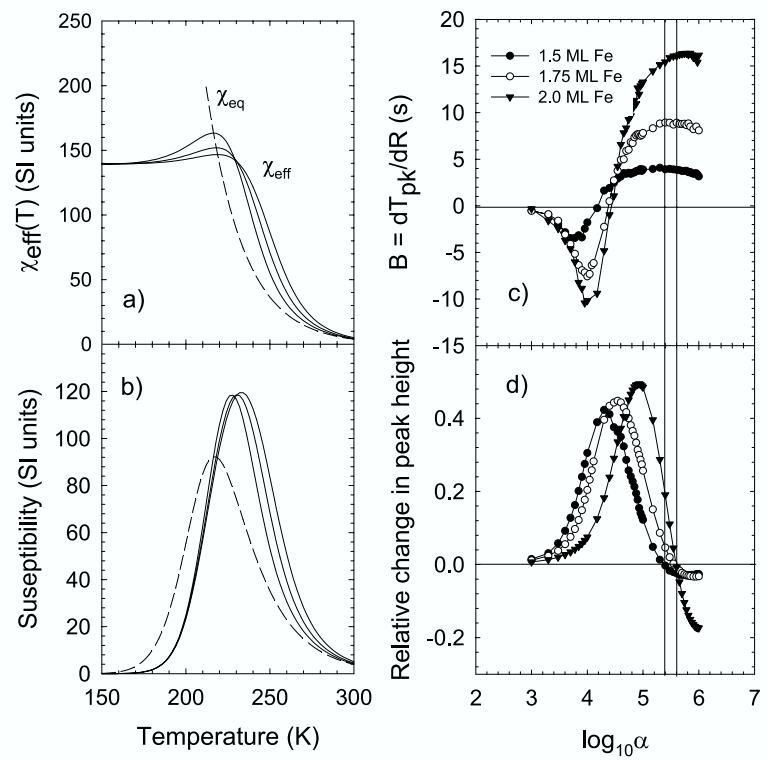

FIG. 5: Calculation of the changes in the susceptibility peak due to domain growth dynamics for a film with $1.75 \mathrm{ML}$ Fe. a) Parameters fit in fig. 2 for the film with a heating rate of 0.30 $\mathrm{K} / \mathrm{s}$ are used to calculate $\chi_{e q}$ directly (dashed line), and $\chi_{e f f}$ through the integration of eq.(13) (solid lines). The heating rates are (left to right) $0.20,0.40$ and $0.70 \mathrm{~K} / \mathrm{s}$. The single adjustable parameter $\alpha=10^{5.5}$. b) The results in part a) are multiplied by the dynamic prefactor in eq.(6) to simulate the measured susceptibility. c) Calculations of the susceptibility as a function of $R$, such as those in part b), are used to find the linear dependence of $T_{p k}$ on $R$ as as function of $\alpha$. d) Calculations of the susceptibility as a function of $R$, such as those in part b), are used to find the relative change in the peak amplitude as a function of $\alpha$.

thicknesses of $1.5,1.75$ and 2.0 ML Fe. As was previously discussed, for small values of $\alpha$, the characteristic temperature for the freezing of the domain density, $T_{n}$, is much less than that for the pinning of the domain wall segments, $T_{p i n}$, and since any changes in domain density dynamics with $R$ cannot be observed, $B \approx 0$. For $\alpha$ i $10^{5}, T_{p i n}<<T_{n}$, and $T_{p k}$ depends upon $R$ through a relatively constant, non-zero linear coefficient $B$. In the intermediate range of $\alpha, T_{p i n} \approx T_{n}$ and the two dynamic mechanisms interact in a complex manner that gives a strong sensitivity of $B$ to the value of $\alpha$.

The two vertical lines on fig. $5 \mathrm{c}$ and $5 \mathrm{~d}$ highlight the region near $\alpha=10^{5.5}$. In this region, $B$ is positive and relatively insensitive to $\alpha$, and the peak height depends only weakly on the heating rate, in agreement with the exper- imental data. These constraints determine the value of $\alpha$ for these films. The corresponding calculated values of $B$ taken from fig. $5 \mathrm{c}$ are appended to Table I, under the heading "B calc.". It can be seen that this single value of the single adjustable parameter $\alpha$ gives good quantitative agreement with "B fit" (within limits of uncertainty) determined from the independent analysis of the plots of $T_{p k}$ in fig. 2.

These results are strong support for the identification of the dynamics of the domain density as the cause of the gradual rise in $T_{p k}$ at large heating rates. These findings validate the model presented in appendix A, where the domain density changes by domain growth driven by dipole interactions, but ultimately hindered by the same actviated domain wall pinning as the local movement of domain walls in response to an applied field. The value of $\alpha \sim 10^{5.5}$ derived from the analysis demonstrates that the domain density relaxes at an intermediate time scale, that is $10^{5.5}$ times slower than the time scale for local pinning of a segment of a domain wall, but much faster than that required for the relaxation of topological defects in the domain pattern.

\section{CONCLUSIONS}

Magnetic susceptibility measurements of $\mathrm{Fe} / 2 \mathrm{ML}$ $\mathrm{Ni} / \mathrm{W}(110)$ films show the effects of three separate magnetic relaxation mechanisms that span a wide range of fundamental time scales. Two of these are readily apparent and have been studied previously. Field-driven oscillatory movement of existing domain walls has a fundamental time scale of $10^{-9} \mathrm{~s}$ and is pinned by structural defects in the film. This dynamical response cuts off the susceptibility below a pinning temperature $T_{p i n}$ and gives the ac susceptibility its characteristic shape. Topological domain pattern defects that persist after quenching from a delocalized phase are removed on a fundamental time scale of $10^{0} \mathrm{~s}$, and cause large ( 25 to $40 \mathrm{~K}$ ) shifts in the entire susceptibility curve when it is measured at a slow heating rate $(R \leq 0.10 \mathrm{~K} / \mathrm{s})$. This shift is to lower temperature as $R$ is increased. Through a systematic study of these effects, it has been possible in the present article to isolate and study a third relaxation mechanism at an intermediate time scale. This is the dipole-driven changes in domain density that create a smaller shift of the ac susceptibility curve to higher temperature as $R$ is increased $(R \geq 0.20 \mathrm{~K} / \mathrm{s})$. The increase in characteristic times of the mechanisms reflects the increase of the size of feature upon which each mechanism works - from the magnetic response of individual Barkhausen steps, to the correlated fluctutations required to drive the growth of domains, to the even more complex rearrangements needed to resolve topological defects involving multiple domains.

A simple activated relaxation model of domain density dynamics provides a quantitative description of 22 susceptibility traces measured at different heating rates 
on films with three different thicknesses, using a single value of a single adjustable parameter. This parameter is $\alpha$, the proportionality between the relaxation time for local domain wall pinning and domain density changes, $\tau_{n}(T)=\alpha \tau_{p}(T)$. The functional form of this relation gives strong support for three features of the model. Most fundamentally, it confirms that the changes in domain density in the experiment are driven by the departure of $n(T)$ from $n_{e q}(T)$ in the absence of a magnetic field, that is by long range dipole forces. Second, that the same structural defects that pin local domain wall movement limit the growth of domains. Finally, that the nucleation of domains is not a limiting factor in changing the domain density in these experiments, and can be neglected. This could be because the nucleation energy is less than the pinning energy, or due to the magnetic history of the films. Since all films are grown at high temperature, they start out with a large density of domains. Upon cooling, the domain density decreases by shrinking the domains, but may leave behind small nuclei which are not eliminated by the weak dipole forces $\frac{12}{\underline{1}}$ Upon heating, growth can occur at the existing nuclei.

The quantitative value $\alpha \approx 10^{5.5}$ confirms that the domain density relaxes on an intermediate time scale. The separation of the three relaxation mechanisms by orders of magnitude permits them to be studied independently by appropriately designed experiments. In particular, changes in the domain density are found to occur independently from the resolution of topological defects, both because of the very different time scales, and the unrelated activation energies. This statement does not mean that structural defects do not affect the local evolution of domain walls in the topological defects, but rather that they are not the limiting factor in their dynamics. These findings are in essential agreement with microscopy studies. The very long relaxation times for topological defects permits them to be imaged, and spatial Fourier transforms of images show a loss of orientational order. The much quicker changes in stripe density, however, cannot be followed, except through the sudden disapperance of individual stripe domains between image line-scans $\frac{6.13}{\underline{6}}$

The present experiments have many similarities to simulations by Bromley et al. 22 These authors also identify three dynamical regimes with different time scales for the relaxation of the magnetic state of Ising spins on a square lattice. There are also important differences, since the simulations study relaxation from a magnetically saturated state at constant temperature, rather than quenching and heating at (almost) zero field. Nonetheless, the initial, fast relaxation of the magnetization in the simulations, driven by Zeeman energies once the field is removed, is clearly analogous to the field-driven domain wall oscillation in the current experiments. Similarly, the subsequent growth and connection of domain segments to form stripes on an intermediate time scale is very reminescent of the model used in the present article for the change the domain density. In the simulations, this process is driven both by dipole interactions and a residual non-zero magnetization. Finally, Bromley et al $\stackrel{22}{\text { find }}$ that topological defects form boundaries separating regions of different stripe orientation, and require a very long time to relax. This is qualtitatively similar to the relaxation of topological defects observed indirectly here. A comparative analysis should not be pressed too far, since the simulations are for very narrow Ising domain walls and do not include a mechanism for domain wall pinning.

\section{ACKNOWLEDGEMENTS}

We are thankful for the continuing technical assistance of M. Kiela, and for insightful discussions with J.P. Whitehead and A.B. MacIsaac. This work was supported by the Natural Sciences and Engineering Research Council of Canada.

\section{Appendix A}

In this appendix, a relaxation equation for changes in the domain density is developed. Assume that the long domains characteristic of the stripe phase are aligned along the y-axis, and that the measurement samples an area of dimension $L_{x} \times L_{y}$. If the $i^{t h}$ domain segment has length $\mathcal{L}_{i}$, then the average domain density is the total domain perimeter divided by the sample area:

$$
n=\frac{1}{L_{x} L_{y}} \sum_{i} 2 \mathcal{L}_{i}
$$

The rate of change of the domain density is then

$$
\frac{d n}{d t}=\frac{2}{L_{x} L_{y}} \sum_{i} \frac{d \mathcal{L}_{i}}{d t}=\frac{2}{L_{x} L_{y}} \sum_{i} 2 v_{i} .
$$

$v_{i}$ is the velocity of the growing (or contracting) tip of the domain segment. The factor of 2 enters because the length of a domain segment changes at both ends.

Due to pinning, the activated average velocity is ${ }^{29}$

$$
v=v_{0} \exp \left(\frac{-\left(E_{\text {pin }}-E_{\text {dipole }}\right)}{k T}\right) .
$$

The fundamental speed $\left|v_{0}\right|$ is given by the product of the Barkhausen step size $s$ and the "attempt" frequency $\nu_{0}=1 / \tau_{0 p}$. The structural defects that pin the domain walls and retard the growth of the domain segments are the same ones that pin the domain walls and retard the change in the width of the domains when an ac field is applied. Therefore, $E_{p i n}$ and $\tau_{0 p}$ are the same quantities in eq.(44) and (A3). Given that these pinning energies are of order $10^{3} \mathrm{~K}$, and that the dipole energy $E_{\text {dipole }}$ driving domain formation is of order $10^{0} \mathrm{~K}$, the latter can be neglected in eq. 
pinning energies can be represented by an average energy $E_{\text {pin }}$, then the distribution of domain wall velocities $v_{i}$ can be represented by the average $v$.

The sum $\frac{1}{L_{x} L_{y}} \sum_{i} 2$ represents the density of active growth fronts. In a relaxation approximation, the density of active growth fronts is proportional to the total density of growth fronts, $\rho$, times the deviation of the number of domain walls from its equilibrium value, $\left(N-N_{e q}\right)$. The total density of growth fronts includes the ends of existing domain segments as well as latent domains nucleated at defects that may grow if the conditions are favourable. This model does not include an explicit nucleation energy, but rather assumes that, upon cooling, the weak dipole energy differences can reduce the domains centred on the nucleation centres to a size such that the domain perimeter is negligible compared to that of the remaining domain segments, but cannot completely remove them. This assumption is supported by microsopy studies of domain formation 12

Putting these factors together,

$$
\frac{d n}{d t}=-\frac{2 \beta s \rho L_{x}}{\tau_{0 p}} \exp \left(\frac{-E_{p i n}}{k T}\right)\left(n-n_{e q}\right) .
$$

The proportionality constant, $\beta$, between the number of active growth sites and $\rho\left(N-N_{e q}\right)$, is difficult to derive. It is related to how strongly the driving dipole energy depends upon the number of domain walls. To the extent that dipole energies are "small" and depend only logarithmically on the number of domains, $\beta$ is expected to be "small" and produce a long relaxation time. The factor $1 /\left(2 \rho L_{x}\right)=\xi$, the average separation of growth sites in the y direction. Finally, the differential equation describing the relaxation of the domain density is

$$
\frac{d n(T)}{d t}=\frac{-1}{\tau_{n}(T)}\left(n(T)-n_{e q}(T)\right)
$$

where $\tau_{n}=\alpha \tau_{p}$, with $\alpha=\xi /(s \beta)$.

\section{Appendix B}

To find how the temperature of the peak of the susceptibility, $T_{p k}$, depends upon the heating rate, it is first necessary to find an expression for $T_{p k}$. Starting from eq.(11), the derivative $\frac{\partial \chi}{\partial T}$ is set to zero to yield the implicit relation

$$
\frac{2 E_{p i n}}{k T_{p k}^{2}} \frac{\omega^{2} \tau_{p}^{2}\left(T_{p k}\right)}{1+\omega^{2} \tau_{p}^{2}\left(T_{p k}\right)}=\left.\frac{1}{n\left(T_{p k}\right)} \frac{\partial n(T)}{\partial T}\right|_{T=T_{,}}
$$

Equation 13 is substituted in for $\frac{\partial n}{\partial T}$, recalling the relation $\tau_{n}(T)=\alpha \tau_{p}(T)$. This gives

$$
\frac{2 E_{p i n}}{k T_{p k}^{2}} \frac{R \alpha}{\omega} \frac{\omega^{3} \tau_{p}^{3}\left(T_{p k}\right)}{1+\omega^{2} \tau_{p}^{2}\left(T_{p k}\right)}=\frac{n_{e q}\left(T_{p k}\right)}{n\left(T_{p k}\right)}-1
$$

As was discussed in section IIC, $T_{p k}$ will depend on the relaxation of the domain density only when $T_{n}>T_{\text {pin }}$. In this case $\omega^{2} \tau_{p i n}^{2}\left(T_{p k}\right)<<1$ and can be neglected in the denominator of the dynamical factor. A further consequence is that near $T_{p k}, n$ is substantially less than $n_{e q}$, as can be seen in fig. 5 a and 5 b. Using these approximations gives as result that is not valid in the limit of very small $R$ :

$$
\frac{2 E_{p i n}}{k T_{p k}^{2}} \frac{R \alpha}{\omega} \omega^{3} \tau_{p}^{3}\left(T_{p k}\right) \approx \frac{n_{e q}\left(T_{p k}\right)}{n\left(T_{p k}\right)} .
$$

To find the implicit derivative $\frac{\partial T_{p k}}{\partial R}$ of the left hand side of this equation is straight forward. For the right hand side,

$$
\begin{aligned}
& \frac{\partial}{\partial R}\left(\frac{n_{e q}\left(T_{p k}\right)}{n\left(T_{p k}\right)}\right)=\left(\frac{n_{e q}\left(T_{p k}\right)}{n\left(T_{p k}\right)}\right) \times \\
& \quad \times\left(\kappa-\left.\frac{1}{n\left(T_{p k}\right)} \frac{\partial n(T)}{\partial T}\right|_{T=T_{p k}}\right)\left(\frac{\partial T_{p k}}{\partial R}\right),
\end{aligned}
$$

where the ansatz in eq.(3) has been used.

Substituting in this equation from eq. (B1) and (B3), and setting the derivatives of the left and right hand side of eq.(B3) equal, yields after rearrangement

$$
1 \approx\left(\frac{\partial T_{p k}}{\partial R}\right)\left(\frac{2}{T_{p k}}+\frac{3 E_{p i n}}{k T_{p k}^{2}}\left(1-\frac{2}{3} \omega^{2} \tau_{p}^{2}\left(T_{p k}\right)\right)+\kappa\right) R .
$$

Using once again the condition $\omega^{2} \tau_{\text {pin }}^{2}\left(T_{p k}\right)<<1$ gives the final estimate

$$
\frac{\partial T_{p k}}{\partial R} \approx \frac{1}{R\left(\frac{2}{T_{p k}}+\frac{3 E_{p i n}}{k T_{p k}^{2}}+\kappa\right)} .
$$

* Electronic address: venus@physics.mcmaster.ca

1 C. Sagui and R.C. Desai, Phys. Rev. E 49, 2225 (1994).

2 S.A. Kivelson, I.P. Bindless, E. Frandkin, V. Oganesyan, J.M. Tranquada, A. Kapitulnik, and C. Howard, Rev. Mod. Phys. 75, 1201 (2003).

${ }^{3}$ K. De'Bell, A.B. MacIsaac, and J.P. Whitehead, Rev. Mod.
Phys. 72, 225 (2000).

${ }^{4}$ R. Allenspach and A. Bischof, Phys. Rev. Lett. 69, 3385 (1992).

${ }^{5}$ D. Venus, C.S. Arnold and M. Dunlavy, Phys. Rev. B 60, 9607 (1999).

${ }^{6}$ O. Portmann, A. Vaterlaus, and D. Pescia, Nature (Lon- 
don) 422, 701 (2003).

7 C. Won, Y.Z. Wu, J. Choi, W. Kim, A. Scholl, A. Doran, T. Owens, J. Wu, X.F. Jin, H.W. Zhoa, and Z.Q. Qui, Phys. Rev. B 71, 224429 (2005).

8 M. Speckmann, H.P. Oepen and H. Ibach, Phys. Rev. Lett. 75, 2035 (1995).

9 A. Vaterlaus, C. Stamm, U. Maier, M.G. Pini, P. Politi, and D. Pescia, Phys. Rev. Lett. 84, 2247 (2000).

10 A. Berger and H. Hopster, Phys. Rev. Lett. 76, 519 (1996).

11 P.J. Metaxas, J.P Jamet, A. Mougin, M. Cormier, J. Ferré, V. Baltz, B. Rodmacq, B. Dieny, and R.L. Stamps, Phys. Rev. Lett. 99, 217208 (2007).

12 N. Saratz, T. Michlmayr, O. Portmann, U. Ramsberger, A. Vaterlaus and D. Pescia, J. Phys. D 40, 1268 (2007).

13 O. Portmann, A. Vaterlaus, and D. Pescia, Phys. Rev. Lett. 96, 047212 (2006).

14 M.J. Dunlavy and D. Venus, Phys. Rev. B 62, 5786 (2000).

15 N. Abu-Libdeh and D. Venus, Phys. Rev. B 80, 184412 (2009).

16 W.J.M. de Jonge, P.J.H. Bloemen and F.J.A. Broeder, in Ultrathin Magnetic Structures I, edited by J.A.C. Bland and B. Heinrich (Springer, Berlin, 1994).

17 Y. Yafet and E.M. Gyorgy, Phys. Rev. B 38, 9145 (1988).

18 A.B. Kashuba and V.L. Pokrovsky, Phys. Rev. B 48, 10335
(1993).

19 A. Abanov, V. Kalatsky, V.L. Pokrovsky, and W.M. Saslow, Phys. Rev. B 51, 1023 (1995).

20 I. Booth, A.B. MacIsaac, J.P. Whitehead, and K. De'Bell, Phys. Rev. Lett. 75, 950 (1995).

21 A.D. Stoycheva and S.J. Singer, Phys. Rev. Lett. 84, 4657 (2000).

22 S.P. Bromley, J.P. Whitehead, K. De'Bell and A.B. MacIsaac, J. Magn. Magn. Mater. 264, 14 (2003).

23 S.A. Cannas, M.F. Michelon, D.A. Stariolo and F.A. Tamarit, Phys. Rev. E 78, 051602 (2008).

24 D. Venus and M.J. Dunlavy, J. Magn. Magn. Mater. 260, 195 (2003).

25 H.L. Johnston, C.S. Arnold and D. Venus, Phys. Rev. B 55, 13221 (1997).

26 C.S. Arnold, H.L. Johnston and D. Venus, Phys. Rev. B 56, 8169 (1997).

27 N. Abu-Libdeh, Ph.D. thesis, McMaster University (2010).

28 C.S. Arnold, M.J. Dunlavy and D. Venus, Rev. Sci. Instrum. 68, 4212 (1997).

29 J. Ferré in Spn Dynamics in Confined Magnetic Structures $I$, edited by B. Hillebrands and K. Ounadjela (Springer, New York, 2002), p. 127. 